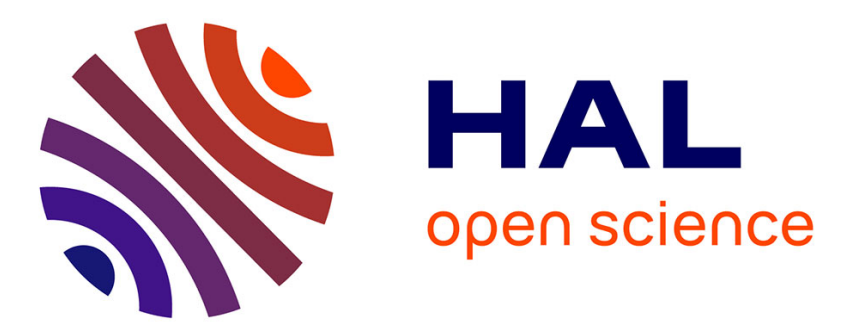

\title{
A comparative study of cavitation models in a Venturi flow
}

\author{
Boris Charrière, Jean Decaix, Eric Goncalves da Silva
}

\section{To cite this version:}

Boris Charrière, Jean Decaix, Eric Goncalves da Silva. A comparative study of cavitation models in a Venturi flow. European Journal of Mechanics - B/Fluids, 2015, 49, p287-297. 10.1016/j.euromechflu.2014.10.003 . hal-01084572

HAL Id: hal-01084572

https://hal.science/hal-01084572

Submitted on 19 Nov 2014

HAL is a multi-disciplinary open access archive for the deposit and dissemination of scientific research documents, whether they are published or not. The documents may come from teaching and research institutions in France or abroad, or from public or private research centers.
L'archive ouverte pluridisciplinaire HAL, est destinée au dépôt et à la diffusion de documents scientifiques de niveau recherche, publiés ou non, émanant des établissements d'enseignement et de recherche français ou étrangers, des laboratoires publics ou privés. 


\title{
A comparative study of cavitation models in a Venturi flow
}

\author{
Boris Charrière $^{\mathrm{a}}$, Jean Decaix ${ }^{\mathrm{b}}$, Eric Goncalvès ${ }^{\mathrm{a}, *}$ \\ ${ }^{a}$ LEGI - University of Grenoble, 1025 rue de la Piscine, 38400 St Martin d'Heres, France \\ ${ }^{b}$ University of Applied Sciences, Western Switzerland, CH-1950 Sion
}

\begin{abstract}
This paper presents a numerical study of an aperiodic cavitation pocket developing in a Venturi flow. The mass transfer between phases is driven by a void ratio transport equation model. A new free-parameter closure relation is proposed and compared with other formulations. The re-entrant jet development, void ratio profiles and pressure fluctuations are analyzed to discern results accuracy. Comparisons with available experimental data are done and good agreement is achieved.
\end{abstract}

Keywords: Cavitation, Mass transfer, Homogeneous model, RANS simulation

\section{Nomenclature}

$\begin{array}{ll}c & \text { speed of sound } \\ C_{p}, C_{v} & \text { thermal capacities } \\ E & \text { total energy } \\ e & \text { internal energy }\end{array}$

${ }^{*}$ Corresponding author

Email address: eric.goncalves@legi.grenoble-inp.fr (Eric Goncalvès ) 


\begin{tabular}{|c|c|}
\hline$k$ & turbulent kinetic energy \\
\hline$P$ & static pressure \\
\hline$P_{v a p}$ & vapour pressure \\
\hline$P_{r}, P_{r t}$ & molecular and turbulent Prandtl numbers \\
\hline$Q$ & total heat flux \\
\hline$R e_{L}$ & Reynolds number based on the length $\mathrm{L}$ \\
\hline$T$ & temperature \\
\hline$u, v$ & velocity components \\
\hline$V$ & vector velocity \\
\hline$w$ & conservative variables \\
\hline$Y$ & mass fraction of gas \\
\hline$\dot{m}$ & mass transfer \\
\hline$\alpha$ & void fraction \\
\hline$\gamma$ & ratio of thermal capacities \\
\hline$\varepsilon$ & dissipation rate \\
\hline$\lambda, \lambda_{t}$ & molecular and turbulent thermal conductivity \\
\hline$\mu, \mu_{t}$ & molecular and eddy viscosity \\
\hline$\rho$ & density \\
\hline$\rho_{I}$ & interfacial density \\
\hline$\sigma$ & cavitation number \\
\hline$\tau$ & total stress tensor \\
\hline$\omega$ & specific dissipation \\
\hline()$_{l}$ & liquid value \\
\hline()$_{v}$ & vapour value \\
\hline()$^{v}$ & viscous \\
\hline
\end{tabular}




\section{Introduction}

Cavitation is the formation of vapour cavities within a given liquid due to pressure drop. It may be observed in various engineering systems such as hydraulic constructions, aeronautics, aerospace, power systems and turbomachinery. The cavitation development may be the origin of several negative effects, such as noise, vibrations, performance alterations, erosion and structural damages. This makes cavitation an important issue in design and operation, which should be controlled, or at least well understood.

Among the cavitation types that may develop, partial cavitation pockets are often observed in hydraulic machines and is known to be responsible for severe damage. Cavitating venturis are one of the simplest case to study such cavities, both experimentally and numerically. This kind of cavitation is characterized by a partial vapour cavity that detaches from the solid body and extends downstream with the existence of a re-entrant jet. The dynamic of these cavitation sheets, the interaction between cavitation and turbulence, the behaviour of the turbulent boundary layer are not yet well known and understood.

Although the numerical modelling of such cavitation has received a great deal of attention, it is still a very difficult and challenging task to simulate such complex unsteady two-phase flow with an acceptable accuracy. Cavitating flows are challenging to model, since they are turbulent with a complex 
interaction with two-phase structures, highly dynamic and involve non equilibrium thermodynamic states. Several numerical models have been developed to investigate such cavitating flows, especially with one-fluid ReynoldsAveraged Navier-Stokes (RANS) solvers. The homogeneous mixture model treats the cavitating flows as a mixture of two fluids behaving as one. These models are based on the assumption of local kinematic equilibrium between phases (the local velocity is the same for both phases), local thermal and mechanic equilibrium between the two components (local temperature and pressure equality between phases). These models are composed by three conservation laws for mixture quantities (mass, momentum and total energy). This model cannot reproduce strong thermodynamic non equilibrium effects but, because of its simplicity, it is often used for numerical simulations $[1,2,3,4,5,6,7,8]$.

By assuming that one pure phase is on a metastable state, a supplementary mass equation or void fraction equation is added. Various formulations of four-equation model have been expressed. A very popular formulation has been developed to simulate turbulent cavitating flows $[9,10,11,12,13,14]$. The main difficulty is related to the formulation of the source term and the tunable parameters involved for the vaporization and condensation processes. Moreover, these models are not thermodynamically well-posed [15]. Another popular model devoted to ebullition problems uses a relaxation term (Homogeneous Relaxation Model). The source term involves a relaxation time estimated from experimental data [16] or with an optimization problem on the mixture entropy [17]. An original formulation was recently proposed for the mass transfer between phases assuming its proportionality with the diver- 
gence of the mixture velocity. This model was validated on various inviscid and turbulent applications $[18,19]$.

The present work is devoted to the numerical study of a partial cavitation pocket appearing on a venturi geometry. For this test case, a transitional behaviour is observed between a stable pocket and a periodic cycles pocket. A particular emphasis is placed on the comparison of various void ratio transport equation models and their ability to capture the re-entrant jet phenomenon. An in-house finite-volume code solving a four-equation RANS compressible system was developed [19]. A new cavitation model is investigated using a mixture of stiffened gas equation of state (EOS). The formulation does not involve any tunable parameter. Validation and comparisons are done with experimental measurements (time-averaged void ratio and velocity profiles, RMS wall pressure fluctuations). A comparison is proposed with OpenFOAM simulations in which the Kunz's void ratio transport equation model is considered. The opensource software OpenFOAM was used and validated in cavitating flows by various authors [20, 21, 22].

In this paper, we will first review the theoretical formulation, including physical models, equation of state and elements of the numerical methods. This is followed by sets of results on a Venturi geometry and discussions.

\section{The LEGI's numerical tool}

The code is based on the solving of the one-fluid compressible RANS system with transport-equation turbulence models. 


\subsection{Reynolds-Averaged Navier-Stokes compressible equations}

The compressible one-fluid RANS equations are used, coupled with a oneor two-equation turbulence model. For low Mach number applications, an inviscid preconditioner is introduced. These equations can be expressed as:

$$
\begin{gathered}
P_{c}^{-1} \frac{\partial w}{\partial t}+\operatorname{div}\left(F_{c}-F_{v}\right)=S \\
w=\left(\begin{array}{c}
\rho \\
\rho V \\
\rho E \\
\alpha \\
\rho k \\
\rho \Psi
\end{array}\right) ; F_{c}=\left(\begin{array}{c}
\rho V \\
\rho V \otimes V+p \overline{\bar{I}} \\
(\rho E+p) V \\
\alpha V \\
\rho k V \\
\rho \Psi V
\end{array}\right) ; F_{v}=\left(\begin{array}{c}
0 \\
\overline{\overline{\tau^{v}}}+\overline{\overline{\tau^{t}}} \\
\left(\overline{\overline{\tau^{v}}}+\overline{\overline{\tau^{t}}}\right) \cdot V-Q^{v}-Q^{t} \\
0 \\
\left(\mu+\mu_{t} / \sigma_{k}\right) \operatorname{grad} k \\
\left(\mu+\mu_{t} / \sigma_{\Psi}\right) \operatorname{grad} \Psi
\end{array}\right)
\end{gathered}
$$

where $w$ denotes the conservative variables and the void ratio, $F_{c}$ and $F_{v}$ the convective and viscous flux densities and $S$ the source terms, which concern the void ratio equation and the turbulent transport equations. The expression of the preconditioning matrix $P_{c}$ is given in [19]. $k$ is the mixture turbulent kinetic energy (TKE) and $\Psi$ is a mixture turbulent variable. In multiphase flow, the divergence of the fluctuating phase velocity is not zero [23]. Therefore, supplementary terms appear in the mixture TKE equation (pressure-dilation term, dilatational dissipation rate), which are not taken into account in the present paper.

The exact expression of the eddy-viscosity $\mu_{t}$ and the source terms depends on the turbulence model as well as constants $\sigma_{k}$ and $\sigma_{\Psi}$.

The total stress tensor $\overline{\bar{\tau}}$ is evaluated using the Stokes hypothesis, Newton's 
law and the Boussinesq assumption. The total heat flux vector $Q$ is obtained from the Fourier law involving a turbulent thermal conductivity $\lambda_{t}$ with the constant Prandtl number hypothesis.

$$
\begin{aligned}
& \overline{\bar{\tau}}=\overline{\overline{\tau^{v}}}+\overline{\overline{\tau^{t}}}=\left(\mu+\mu_{t}\right)\left[\left(\operatorname{grad} V+(\operatorname{grad} V)^{t}\right)-\frac{2}{3}(\operatorname{div} V) \overline{\bar{I}}\right]+\frac{2}{3} \rho k \overline{\bar{I}} \\
& Q=Q^{v}+Q^{t}=-\left(\lambda+\lambda_{t}\right) \operatorname{grad} T \quad \text { with } \quad \lambda_{t}=\frac{\mu_{t} C_{p}}{P_{r t}}
\end{aligned}
$$

In pure phases, the viscosity is assumed to be constant. The mixture viscosity is defined as the arithmetic mean of the liquid and vapour viscosities:

$$
\mu(\alpha)=\alpha \mu_{V}+(1-\alpha) \mu_{L}
$$

The mixture thermal conductivity $\lambda$ is also defined as the arithmetic mean of the liquid and vapour values:

$$
\lambda(\alpha)=\alpha \frac{\mu_{V} C_{p_{V}}}{P_{r_{V}}}+(1-\alpha) \frac{\mu_{L} C_{p_{L}}}{P_{r_{L}}}
$$

The turbulent Prandtl number $P_{r t}$ is set to 1.

To close the system, an equation of state (EOS) is necessary to link the pressure and the temperature to the internal energy and the density. For the pure phases, we used the convex stiffened gas EOS:

$$
\begin{aligned}
P(\rho, e) & =(\gamma-1) \rho(e-q)-\gamma P_{\infty} \\
P(\rho, T) & =\rho(\gamma-1) C_{v} T-P_{\infty} \\
T(\rho, h) & =\frac{h-q}{C_{p}}
\end{aligned}
$$

where $\gamma=C_{p} / C_{v}$ is the heat capacity ratio, $C_{p}$ and $C_{v}$ are thermal capacities, $q$ the energy of the fluid at a given reference state and $P_{\infty}$ is a constant reference pressure. 


\subsection{A void ratio transport equation}

A void ratio equation can be expressed as [24]:

$$
\begin{array}{r}
\frac{\partial \alpha}{\partial t}+\operatorname{div}(\alpha V) \quad=(K+\alpha) \operatorname{div} V+\frac{\dot{m}}{\rho_{I}} \\
K=\left(\frac{\rho_{l} c_{l}^{2}-\rho_{v} c_{v}^{2}}{\frac{\rho_{l} c_{l}^{2}}{1-\alpha}+\frac{\rho_{v} c_{v}^{2}}{\alpha}}\right) \quad ; \quad \rho_{I}=\left(\frac{\frac{c_{v}^{2}}{\alpha}+\frac{c_{l}^{2}}{1-\alpha}}{\frac{\rho_{l} c_{l}^{2}}{1-\alpha}+\frac{\rho_{v} c_{v}^{2}}{\alpha}}\right)
\end{array}
$$

where $\dot{m}$ is the mass transfer between phases and $\rho_{I}$ the interfacial density .

By assuming that the mass transfer is proportional to the divergence of the velocity, it is possible to build a family of models in which the mass transfer $\dot{m}$ is expressed as $[18]$

$$
\dot{m}=\frac{\rho_{l} \rho_{v}}{\rho_{l}-\rho_{v}}\left(1-\frac{c^{2}}{c_{\text {wallis }}^{2}}\right) \operatorname{div} V
$$

where $c_{\text {wallis }}$ is the propagation velocity of acoustic waves without mass transfer [25]. This speed of sound is expressed as a weighted harmonic mean of speeds of sound of each phase:

$$
\frac{1}{\rho c_{\text {wallis }}^{2}}=\frac{\alpha}{\rho_{v} c_{v}^{2}}+\frac{1-\alpha}{\rho_{l} c_{l}^{2}}
$$

A first model was built using the speed of sound associated with a sinusoidal barotropic EOS [18, 19]. In the following, this model will be named 4-equation barotropic model. It involves one tunable parameter $c_{\text {baro }}$ interpreted as the minimum value of the speed of sound in the mixture. For all simulations the value was set to $0.5 \mathrm{~m} / \mathrm{s}$.

\subsection{A new cavitation model}

The new model is based on a mixture of stiffened gas EOS. By assuming the pressure equilibrium between phases, an expression for the pressure can 
be deduced, function of the void ratio $\alpha$ and the vapour mass fraction $Y=$ $\alpha \rho_{v} / \rho$ :

$$
\begin{aligned}
P(\rho, e, \alpha, Y) & =(\gamma(\alpha)-1) \rho(e-q(Y))-\gamma(\alpha) P_{\infty}(\alpha) \\
\frac{1}{\gamma(\alpha)-1} & =\frac{\alpha}{\gamma_{v}-1}+\frac{1-\alpha}{\gamma_{l}-1} \\
q(Y) & =Y q_{v}+(1-Y) q_{l} \\
P_{\infty}(\alpha) & =\frac{\gamma(\alpha)-1}{\gamma(\alpha)}\left[\alpha \frac{\gamma_{v}}{\gamma_{v}-1} P_{\infty}^{v}+(1-\alpha) \frac{\gamma_{l}}{\gamma_{l}-1} P_{\infty}^{l}\right]
\end{aligned}
$$

By assuming the thermal equilibrium between phases, the mixture temperature is expressed as:

$$
\begin{aligned}
T(\rho, h, Y) & =\frac{h_{l}-q_{l}}{C_{p_{l}}}=\frac{h_{v}-q_{v}}{C_{p_{v}}}=\frac{h-q(Y)}{C_{p}(Y)} \\
C_{p}(Y) & =Y C_{p_{v}}+(1-Y) C_{p_{l}}
\end{aligned}
$$

The speed of sound in the mixture can be expressed as a function of the enthalpy of each phase (see Appendix A):

$$
\rho c^{2}=\frac{1}{\gamma-1}\left[\frac{\rho_{v} \rho_{l}}{\left(\rho_{l}-\rho_{v}\right)}\left(h_{v}-h_{l}\right)\right]
$$

Enthalpies of pure phase $h_{l}$ and $h_{v}$ are computed with the mixture temperature $T$.

The mass transfer term is activated when the local pressure $P$ is smaller than the vapour pressure $P_{\text {vap }}$. This model will be named 4-equation SG model. It does not involve any tunable parameter.

\subsection{The turbulence model}

Various turbulence models are considered: the Smith $k-\ell$ model (KL) [26], the one-equation Spalart-Allmaras model (SA) [27] and the Jones-Launder 
$k-\varepsilon$ model (KE) [28]. For a correct simulation of the re-entrant jet, the Reboud eddy-viscosity limiter is added [29, 30, 31]. For comparisons with the OpenFOAM solver, the Menter $k-\omega$ SST model [32] is used, assuming the validity of the Bradshaw assumption in a two-phase turbulent boundary layer.

\subsection{Wall functions}

For the modelling of flow close to the wall, a two-layer wall law approach is used:

$$
\begin{array}{rlrlrl}
u^{+} & =y^{+} & & \text {if } & y^{+}<11.13 \\
u^{+} & =\frac{1}{\kappa} \ln y^{+}+5.25 & \text { if } & y^{+}>11.13 \\
u^{+} & =\frac{u}{U_{\tau}} & ; & y^{+}=\frac{y U_{\tau}}{\nu_{w}} \quad ; & U_{\tau}^{2}=\frac{\tau_{w}}{\rho_{w}}
\end{array}
$$

where $\kappa=0.41$ is the von Karman constant and the subscript 'w' is used for a wall value.

We assume that wall functions are similar in a two-phase flow and in a single-phase flow. For unsteady flows, the existence of a wall law is assumed to be valid at each instant. These assumptions have been studied in [33] and comparisons were proposed with a thin boundary layer approach.

\subsection{Numerics}

The numerical simulations are carried out using an implicit CFD code based on a finite-volume discretization. For the mean flow, the convective flux density vector on a cell face is computed with the Jameson-Schmidt-Turkel scheme [34]. The artificial viscosity includes a second-order dissipation term 
$D_{2}$ and a fourth-order dissipation term $D_{4}$, which involve two tunable parameters $k^{(2)}$ and $k^{(4)}$.

The viscous terms are discretized by a second-order space-centered scheme. For the turbulence transport equations, the upwind Roe scheme [35] is used to obtain a more robust method. The second-order accuracy is obtained by introducing a flux-limited dissipation [36].

Time integration is achieved using the dual time stepping approach and a low-cost implicit method consisting in solving, at each time step, a system of equations arising from the linearization of a fully implicit scheme. The derivative with respect to the physical time is discretized by a second-order formula.

The numerical treatment of boundary conditions is based on the use of the preconditioned characteristic relationships. More details are given in [19].

\section{The OpenFOAM code}

The OpenFOAM code is an open source code distributed by ESI Group. It is based on an orientated object framework [37]. It provides a large variety of RANS turbulence models and cavitation models. For cavitation modelling, two ways are available: either to use an equation of state for the mixture or to use a transport equation for the volume fraction of liquid. The last one is retained for the present simulation.

\subsection{Cavitation model}

The cavitation model reads:

$$
\frac{\partial \alpha_{l}}{\partial t}+u_{j} \frac{\partial \alpha_{l}}{\partial x_{j}}=S
$$


where $\alpha_{l}$ is the volume fraction of liquid and $S$ is the mass source term.

Following Kunz development [10], the source term is expressed as the sum of a vaporisation term $m_{v}$ and a condensation term $m_{c}$ :

$$
S=m_{v}+m_{c}
$$

with:

$$
\begin{aligned}
m_{c} & =\frac{\rho}{\rho_{l} \rho_{v}} C_{c} \frac{\rho_{v}}{t_{\infty}} \alpha_{\text {Llim }}^{2} \frac{\max \left(P-P_{\text {vap }} ; 0\right)}{\max \left(P-P_{v a p} ; 0.01 P_{v a p}\right)} \\
m_{v} & =\frac{\rho}{\rho_{l} \rho_{v}} C_{v} \frac{\rho_{v}}{\frac{1}{2} \rho_{l} U_{\infty}^{2} t_{\infty}} \min \left(P-P_{v a p} ; P_{0}\right)
\end{aligned}
$$

$C_{c}, C_{v}, U_{\infty}$ and $t_{\infty}$ are constant set by the user, whereas $P_{0}$ and $\alpha_{\text {Llim }}$ are included to avoid non physical values. Usually $U_{\infty}$ is set to the freestream value, and $t_{\infty}$ represents a relaxation time not well defined in the literature. For the present computations, the following values are specified:

$$
C_{c}=10 ; C_{v}=8000 ; U_{\infty}=10.8 \mathrm{~m} / \mathrm{s} ; t_{\infty}=0.005 \mathrm{~s}
$$

The model uncertainty should be analyzed using non-intrusive stochastic methods as presented in [38].

\subsection{The turbulence model}

The $k-\omega$ SST model proposed by Menter [32] is used to solve the turbulent kinetic energy and the specific dissipation with the standard values of the different parameters.

\subsection{Numerics}

The time derivatives are computed with the backward second order scheme. Excepted for the volume fraction of liquid, the convective flux are discretised 
with the Total Variation Diminishing (TVD) scheme named 'limitedLinear' specific to OpenFOAM with the parameter set to 1.

Whereas the momentum equation and the Poisson equation are treated implicitly, the equation for the volume fraction of liquid is treated explicitly and separately. To maintain the boundedness of the liquid volume fraction, the Multidimensional Universal Limiter for Explicit Solution (MULES) is used and a counter-gradient is introduced to reduce the diffusion of the interface and enhanced the numerical stability. The convective flux of the transport equation for the volume fraction of liquid is computed with the van Leer scheme.

The set of equation is solved using a prediction-correction approach coupling the SIMPLE and PISO algorithm.

\section{Experimental and numerical parameters}

\subsection{Experimental conditions}

The Venturi was tested in the cavitation tunnel of the CREMHyG (Centre d'Essais de Machines Hydrauliques de Grenoble). It is characterized by a divergence angle of $4^{\circ}$, illustrated in Figure 1 . The edge forming the throat of the Venturi is used to fix the separation point of the cavitation cavity. This geometry is equipped with five probing holes to allow various measurements such as the local void ratio, instantaneous local speed and wall pressure (Figure 1). The velocity is evaluated as the most probable value and the void ratio is obtained from the signal of the double optical probe using a post-processing algorithm. The relative uncertainty on the void ratio measurement was estimated at roughly $15 \%$ [39]. 
The selected operating point is characterized by the following physical parameters [39]:

$U_{\text {inlet }}=10.8 \mathrm{~m} / \mathrm{s}$, the inlet velocity

$\sigma_{\text {inlet }}=\frac{P_{\text {inlet }}-P_{\text {vap }}}{0.5 \rho U_{\text {inlet }}^{2}} \simeq 0.55$, the cavitation parameter in the inlet section

$T_{\text {ref }} \simeq 293 \mathrm{~K}$, the reference temperature

$L_{r e f}=252 \mathrm{~mm}$, the reference length

$R e_{L_{r e f}}=\frac{U_{\text {inlet }} L_{r e f}}{\nu}=2.710^{6}$, the Reynolds number

With these parameters, a cavity length $L$ ranging from $70 \mathrm{~mm}$ to $85 \mathrm{~mm}$ was obtained. The experimental views for this geometry show a relatively stable cavity behaviour (see Fig. 2). The attached cavity length corresponding to the end of the re-entrant jet is around $30-35 \mathrm{~mm}$. For this geometry, no periodic cycles with large shedding were observed.

\subsection{Mesh and numerical parameters}

The grid is a H-type topology. It contains 251 nodes in the flow direction and 62 nodes in the orthogonal direction. A special contraction of the mesh is applied in the main flow direction just after the throat to better simulate the two-phase flow area. The $y^{+}$values of the mesh, at the center of the first cell, vary between 12 and 27 for a non cavitating computation.

Unsteady computations are performed with the dual time stepping method and are started from the non cavitating numerical solution. The numerical parameters are:

- the dimensionless time step, $\Delta t^{*}=\frac{\Delta t U_{\text {inlet }}}{L_{\text {ref }}}=4.8810^{-3}$

- sub-iterations of the dual time stepping method, 100 
- the CFL number, 0.2

- Jacobi iterations for the implicit stage, 15

- the two coefficients of the artificial dissipation, $k^{(2)}=1$ and $k^{(4)}=0.045$.

\section{Computational results}

Various computations were performed by varying the cavitation model and the turbulence model, summarized in Table (1). The goal was to obtain a sheet whose time-averaged attached and total length varied around 30-35 $\mathrm{mm}$ and 75-85 mm, respectively. The time of simulation is between 2 and $3 \mathrm{~s}$.

\subsection{Limitation of the eddy viscosity}

A key point to compute the unsteadiness of the cavitation pocket is linked to the over-production of eddy viscosity by standard turbulence models. Previous simulations based on a three-equation model illustrated the importance of using an eddy-viscosity limiter to capture the re-entrant jet dynamics [30]. In this study, the Reboud limiter is added to the turbulence model. The effect of this limiter using the Spalart-Allmaras is showed in Figure 3 where are plotted the contours of the density gradient modulus (Schlieren-like visualizations). When the turbulent viscosity is reduced by the correction, the length of attached cavity reaches the experimental value around $0.35 \mathrm{~m}$ and vapour clouds appear.

We observed the same effect for other turbulence models and results are not presented. 


\subsection{Turbulence models comparison}

Computations are done using the four-equation barotropic model associated to three turbulence models in which the Reboud limiter is added. All numerical values are obtained by a time-averaged statistical treatment over a simulation time of $2 \mathrm{~s}$.

Fig. 4 presents the void ratio and velocity profiles from stations 3 to 5 . At stations 1 and 2, inside the attached cavity, all simulations provide the same results in close agreement with the experimental data and are not presented. At station 3, the re-entrant jet is observed on the velocity measurement. All simulations indicate a recirculating behaviour with a re-entrant jet extending through half the sheet thickness. For the void ratio profiles, the three simulations give very close results.

At station 4 and 5 the whole of simulation capture the re-entrant jet characterized by negative velocitites close to the wall. As regard to the void ratio profiles, we observe an over-estimation at station 4 using the $k-\phi$ models and a better estimation using the Spalart-Allmaras model. At station 5, the $k-\ell$ turbulence model overestimates a little the length of the cavity.

The dimensionless wall pressure distribution $\frac{P-P_{v a p}}{P_{\text {vap }}}$ is plotted in Figure 5 versus the distance $x-x_{\text {inlet }}$. The first five data are located inside the cavity (where the void ratio and velocity profiles are measured). All models provide a pressure distribution similar to the experimental measurements upstream of the re-compression. 
The Root Mean Square (RMS) wall pressure fluctuations are plotted in Figure 6 versus the distance $x-x_{\text {inlet }}$. The pressure fluctuation is divided by the time-averaged pressure Pav. Experimental data indicate an augmentation of pressure fluctuations at the end of the sheet cavity. All simulations predict a peak of pressure fluctuations located close to the experimental abscissa. The magnitude of the peak is overestimated by the computations, but with the lack of experimental values in this area the comparison cannot be precise. Nevertheless, downstream the cavity, the pressure fluctuations are over-predicted using the Spalart-Allmaras and $k-\varepsilon$ turbulence models. This phenomenon has already been observed in a previous study and can be corrected by modifying the source term in the void ratio equation [19].

The dynamic of cavitation pocket is also studied with the iso-lines of the Qcriterion. Positive values of the Q-criterion, defined as the second invariant of the velocity gradient tensor $\frac{\partial u_{i}}{\partial x_{j}}[40]$,

$$
Q=\frac{1}{2}\left[\left(\frac{\partial u_{i}}{\partial x_{i}}\right)^{2}-\frac{\partial u_{i}}{\partial x_{j}} \frac{\partial u_{j}}{\partial x_{i}}\right]
$$

are used to identify vortices and local rotational areas. A dimensionless quantity is built using the inlet velocity and the reference length. Iso-lines levels vary between 0.005 and 0.1 . The results are illustrated in Figure 7. For all turbulence models, the shear layer creates vortical clouds of cavitation, which are convected by the mean flow. No significant discrepancies on the flow dynamic are noticeable between the three simulations.

To conclude, similar results have been obtained with the three turbulence 
models associated to an eddy viscosity limiter. The re-entrant jet is well estimated but vapour ratio tends to be overestimated especially in the recirculating area (station 3 and 4). The influence of the turbulence model is weak for this partial cavitation pocket.

In the next part, a mixture of stiffened gas EOS is proposed to replace the barotropic EOS and to investigate the cavitation model influence.

\subsection{Cavitation models comparison}

For this study, the Spalart-Allmaras turbulence model is chosen and the two formulations of 4-equation model are compared.

The dynamic of the cavitation sheets is proposed in Figure 8 where the contours of the density gradient modulus are presented at two different times. Both simulations propose similar dynamics and the generation of vapour cloud shedding are clearly illustrated.

A more precise comparison is led by studying void ratio and velocity profiles, which are presented in Figure 9 from stations 3 to 5 . At region of attached cavity sheet (stations 1 and 2), both simulations provide a well estimation of the cavity thickness and results are not presented.

Differences appear at station 3 where the four-equation SG cavitation model provides a better prediction of the void ratio profile characterized by a significant decrease close to the wall. However, by comparison with the experimental data and the four-equation barotropic velocity profiles, this model underestimates the recirculating flow in this area. At stations 4 and 5 the four-equation SG cavitation model also reproduces the re-entrant jet on the 
half bottom of the cavity but it over-predicts the vapour ratio in the remaining part. The wall value of the void ratio is better simulated with the new model.

As it can be observed in Figure 10 both models give a time-averaged wall pressure distribution in good agreement with the experimental data. The Root Mean Square (RMS) wall pressure fluctuations are plotted in Figure 11. Both models predict a similar peak intensity at the same location. It appears that the four-equation SG cavitation model provides a better estimation of the pressure fluctuations decrease in the re-compression area.

This study reveals that the four-equation SG cavitation is well adapted to simulate the behaviour of the cavitation pocket especially in the closure part of the cavity. Moreover, this model presents the large advantage to not involve tunable parameters. In the next part, this model will be used to compare the LEGI's and OpenFOAM softwares.

\subsection{Comparison with OpenFOAM simulations}

Comparisons between the LEGI solver and OpenFOAM are proposed on a similar mesh using the $k-\omega$ SST turbulence model. The considered cavitation models are the 4-equation SG model and the Kunz model, respectively. The time of simulation is about $2 \mathrm{~s}$.

Time-averaged void ratio and velocity profiles are presented in Figure 12 from stations 1 to 5 . Inside the attached cavity sheet, at stations 1 and 2, the both solvers estimate a well cavity thickness and composition. At stations 3 and 
4, the LEGI solver provides a better prediction of the void ratio decrease due to the presence of a mixture in this area. The OpenFOAM computation over-predicts the vapour quantity inside the cavity (95\% instead of $70 \%$ at station 3 and $90 \%$ instead of $30 \%$ at station 3). Moreover, the thickness of the cavity is under-estimated and a pure liquid phase is simulated close to the wall. At station 5, both the cavity thickness and the re-entrant jet are badly simulated by the OpenFOAM software. Although the re-entrant phenomenon is well observed in experiments, the OpenFOAM simulation does not reproduce the recirculation area.

The time-averaged wall pressure distributions are compared in Figure 13. The two models present a different re-compression area respectively located downstream the experimental measures with the LEGI solver and upstream with the OpenFOAM solver. The rate of re-compression is also underestimated by the OpenFOAM solver.

A study of the Root Mean Square (RMS) wall pressure fluctuations is proposed in Figure 14. The peak of fluctuations position is varying among the case. The OpenFOAM solver provides a maximum of fluctuations located upstream the closure area of the cavity sheet $\left(x-x_{i} \approx 0.2\right.$ in experiments), whereas it is predicted downstream by the LEGI solver.

To conclude, the topology of the cavitation pocket marks large discrepancies between the two softwares, especially as regards the re-entrant jet development. Maybe a better calibration of the production and evaporation con- 
stants appearing in the mass transfer formulation should improve the results for this test case.

\section{Conclusion}

An aperiodic partial cavitation pocket has been studied in a $2 \mathrm{D}$ venturi configuration by numerical one-fluid unsteady RANS simulations. Numerical results have been compared with experimental data concerning the void ratio, streamwise velocity, wall pressure and wall pressure fluctuations.

First, calculations have been carried out with an in-house code. The one-fluid RANS equations have been successively coupled with the Smith $k-\ell$, the Spalart-Allmaras, the Jones-Launder $k-\varepsilon$ and the Menter $k-\omega$ SST turbulence models. Two cavitation models using an explicit formulation of the mass transfer between phases have also been compared: the first one based on a barotropic EOS and the second one based on a mixture of stiffened gas EOS. Comparisons revealed that similar results were obtained using different turbulence models when an eddy viscosity limiter is introduced. For this test case, the turbulence model influence is weak. Then, a cavitation model comparison was performed using the Spalart-Allmaras turbulence model. The new four-equation model has improved the re-entrant jet simulation downstream the attached cavity by diminishing the void ratio values close to the wall. A better estimation of the pressure fluctuations is also provided by this model. A very positive point is the free-parameter formulation of the source term, which avoids poisonous calibration problems.

Secondly, simulations has been carried out with the OpenFOAM software us- 
ing another formulation of void ratio transport equation cavitation models. Calculations were performed on a similar mesh using the Menter SST turbulence model. Large discrepancies appeared between the two solvers and the OpenFOAM simulations were not able to reproduce the re-entrant jet phenomenon. Maybe it is due to a calibration problem of the mass transfer model involving two tunable parameters. The new LEGI model is therefore very attractive to simulate such partial cavitation pockets.

Additional works are in progress to pursue comparative analyses between turbulence and cavitation models and to extend the formulation with thermodynamic effects.

\section{Appendix}

Appendix A: the speed of sound in a mixture of stiffened gas

Starting from the usual thermodynamic relation

$$
d e=T d s+\frac{P}{\rho^{2}} d \rho \quad \text { or } \quad d(\rho e)=\rho T d s+h d \rho
$$

And with the differential of $\rho e$ :

$$
d(\rho e)=\left(\frac{\partial \rho e}{\partial \rho}\right)_{P} d \rho+\left(\frac{\partial \rho e}{\partial P}\right)_{\rho} d P
$$

We can obtain the differential of the pressure $P$ :

$$
\left(\frac{\partial \rho e}{\partial P}\right)_{\rho} d P=\rho T d s+\left[h-\left(\frac{\partial \rho e}{\partial \rho}\right)_{P}\right] d \rho
$$

We deduce an expression of the speed of sound:

$$
c^{2}=\left(\frac{\partial P}{\partial \rho}\right)_{s}=\frac{h-\left(\frac{\partial \rho e}{\partial \rho}\right)_{P}}{\left(\frac{\partial \rho e}{\partial P}\right)_{\rho}}
$$


With the stiffened gas EOS, we have:

$$
\begin{aligned}
\left(\frac{\partial \rho e}{\partial P}\right)_{\rho} & =\alpha\left(\frac{\partial \rho_{v} e_{v}}{\partial P}\right)_{\rho}+(1-\alpha)\left(\frac{\partial \rho_{l} e_{l}}{\partial P}\right)_{\rho}=\frac{1}{\gamma-1} \\
\left(\frac{\partial \rho e}{\partial \rho}\right)_{P} & =\frac{\partial}{\partial \rho}\left[\alpha\left(\frac{P}{\gamma_{v}-1}+\rho_{v} q_{v}+\frac{\gamma_{v} P_{\infty}^{v}}{\gamma_{v}-1}\right)+(1-\alpha)\left(\frac{P}{\gamma_{l}-1}+\rho_{l} q_{l}+\frac{\gamma_{l} P_{\infty}^{l}}{\gamma_{l}-1}\right)\right] \\
& =\frac{\rho_{l} h_{l}-\rho_{v} h_{v}}{\rho_{v}-\rho_{v}}
\end{aligned}
$$

Finally, the speed of sound is:

$$
\rho c^{2}=\rho\left(\frac{\partial P}{\partial \rho}\right)_{s}=(\gamma-1)\left[\frac{\rho_{v} \rho_{l}}{\left(\rho_{l}-\rho_{v}\right)}\left(h_{v}-h_{l}\right)\right]
$$


[1] D. Schmidt, C. Rutland, M. Corradini, A fully compressible, twodimensional model of small, high-speed, cavitating nozzles, Atomization and Sprays 9 (1999) 255-276.

[2] T. Liu, B. Khoo, W. Xie, Isentropic one-fluid modelling of unsteady cavitating flow, Journal of Computational Physics 201 (1) (2004) 80108.

[3] T. Barberon, P. Helluy, Finite volume simulation of cavitating flows, Computers \& Fluids 34 (7) (2005) 832-858.

[4] E. Sinibaldi, F. Beux, M. Salvetti, A numerical method for 3D barotropic flows in turbomachinery, Flow Turbulence Combustion 76 (2006) 371381.

[5] S. Schmidt, I. Sezal, G. Schnerr, Compressible simulation of high-speed hydrodynamics with phase change, in: European Conference on Computational Fluid Dynamics ECCOMAS 2006, Delft, The Netherlands, 2006.

[6] W. Xie, T. Liu, B. Khoo, Application of a one-fluid model for large scale homogeneous unsteady cavitation: The modified Schmidt model, Computers \& Fluids 35 (2006) 1177-1192.

[7] E. Goncalves, R. F. Patella, Numerical simulation of cavitating flows with homogeneous models, Computers \& Fluids 38 (9) (2009) 16821696.

[8] M. Bilanceri, F. Beux, M. Salvetti, An implicit low-diffusive HLL scheme 
with complete time linearization: application to cavitating barotropic flows, Computers \& Fluids 39 (2010) 1990-2006.

[9] C. Merkle, J. Feng, P. Buelow, Computation modeling of the dynamics of sheet cavitation, in: $3^{\text {rd }}$ International Symposium on Cavitation CAV1998, Grenoble, France, 1998.

[10] R. Kunz, D. Boger, D. Stinebring, T. Chyczewski, J. Lindau, H. Gibeling, S. Venkateswaran, T. Govindan, A preconditioned Navier-Stokes method for two-phase flows with application to cavitation prediction, Computers \& Fluids 29 (8) (2000) 849-875.

[11] G. Schnerr, J. Sauer, Physical and numerical modeling of unsteady cavitation dynamics, in: 4th Int. Conference on Multiphase Flow ICMF01, New Orleans, USA, 2001.

[12] I. Senocak, W. Shyy, A pressure-based method for turbulent cavitating flow computations, Journal of Computational Physics 176 (2) (2002) 363-383.

[13] A. Singhal, M. Athavale, H. Li, Y. Jiang, Mathematical basis and validation of the full cavitation model, Journal of Fluids Engineering 124 (3) (2002) 617-624.

[14] Y. Utturkar, J. Wu, G. Wang, W. Shyy, Recent progress in modelling of cryogenic cavitation for liquid rocket propulsion, Progress in Aerospace Sciences 41 (2005) 558-608. 
[15] E. Goncalves, R. F. Patella, Constraints on equation of state for cavitating flows with thermodynamic effects, Applied Math. and Computation 217 (2011) 5095-5102.

[16] P. Downar-Zapolski, Z. Bilicki, L. Bolle, J. Franco, The non-equilibrium relaxation model for one-dimensional flashing liquid flow, Int. Journal of Multiphase Flow 22 (3) (1996) 473-483.

[17] P. Helluy, N. Seguin, Relaxation models of phase transition flows, Mathematical Modelling and Numerical Analysis 40 (2) (2006) 331-352.

[18] E. Goncalves, Numerical study of expansion tube problems: Toward the simulation of cavitation, Computers \& Fluids 72 (2013) 1-19.

[19] E. Goncalves, B. Charriere, Modelling for isothermal cavitation with a four-equation model, International Journal of Multiphase Flow 59 (2014) $54-72$.

[20] R. Bensow, G. Bark, Simulating cavitating flows with LES in openfoam, in: V ECCOMAS CFD, Lisbon, Portugal, June 2010.

[21] S. Park, S. Rhee, Computational analysis of turbulent super-cavitating flow around a two-dimensional wedge-shaped cavitator geometry, Computers \& Fluids 70 (2012) 73-85.

[22] Z. Shang, Numerical investigations of supercavitation around blunt bodies of submarine shape, Applied Mathematical Modelling 37 (2013) 8836-8845. 
[23] J. Decaix, E. Goncalves, Compressible effects modelling in turbulent cavitating flows, European J. of Mechanics B/Fluids 39 (2013) 11-31.

[24] R. Saurel, F. Petitpas, R. Abgrall, Modelling phase transition in metastable liquids: application to cavitating and flashing flows, Journal of Fluid Mechanics 607 (2008) 313-350.

[25] G. Wallis, One-dimensional two-phase flow, New York: McGraw-Hill (1967).

[26] B. Smith, A near wall model for the $k-l$ two equation turbulence model, in: AIAA 94-2386, 25 $5^{\text {sh }}$ Fluid Dynamics Conference - Colorado Springs, Colorado, 1994.

[27] P. Spalart, S. Allmaras, A one-equation turbulence model for aerodynamic flows, La Recherche Aérospatiale (1) (1994) 5-21.

[28] W. Jones, B. Launder, The prediction of laminarization with a twoequation model of turbulence, Int. J. Heat and Mass Transfer 15 (1972) $301-314$.

[29] J.-L. Reboud, B. Stutz, O. Coutier, Two-phase flow structure of cavitation: experiment and modelling of unsteady effects, in: $3^{\text {rd }}$ International Symposium on Cavitation CAV1998, Grenoble, France, 1998.

[30] E. Goncalves, Numerical study of unsteady turbulent cavitating flows, European Journal of Mechanics B/Fluids 30 (1) (2011) 26-40.

[31] J. Decaix, E. Goncalves, Time-dependent simulation of cavitating flow 
with $k-\ell$ turbulence models, Int. Journal for Numerical Methods in Fluids 68 (2012) 1053-1072.

[32] F. Menter, Two-equation eddy-viscosity turbulence models for engineering applications, AIAA Journal 32 (8) (1994) 1598-1605.

[33] E. Goncalves, J. Decaix, Wall model and mesh influence study for partial cavities, European Journal of Mechanics B/Fluids 31 (1) (2012) 12-29.

[34] A. Jameson, W. Schmidt, E. Turkel, Numerical solution of the Euler equations by finite volume methods using Runge-Kutta time stepping schemes, in: AIAA Paper 81-1259, 1981.

[35] P. Roe, Approximate Riemann solvers, parameters vectors, and difference schemes, Journal of Computational Physics 43 (1981) 357-372.

[36] S. Tatsumi, L. Martinelli, A. Jameson, Flux-limited schemes for the compressible Navier-Stokes equations, AIAA Journal 33 (2) (1995) 252261.

[37] H. Weller, G. Tabor, H. Jasak, C. Fureby, A tensorial approach to computational continuum mechanics using objected-oriented techniques, Computers in Physics 12 (1998) 620-631.

[38] M. Rodio, P. Congedo, Robust analysis of cavitating flows in Venturi tube, European J. of Mechanics B/Fluids in press.

[39] S. Barre, J. Rolland, G. Boitel, E. Goncalves, R. F. Patella, Experiments and modelling of cavitating flows in Venturi: attached sheet cavitation, European Journal of Mechanics B/Fluids 28 (2009) 444-464. 
[40] J. Hunt, C. Wray, P. Moin, Eddies, streams, and convergence zones in turbulent flows, Tech. rep., Center for Turbulence Research, CTR-S88 (1988). 
Table 1: Unsteady computations, $4^{\circ}$ Venturi.

\begin{tabular}{lllc}
\hline cav. model & turb. model & $\sigma_{\text {inlet }}$ & attached and total sheet length $(\mathrm{m})$ \\
\hline 4-eqt baro & $\mathrm{SA}$ & 0.59 & $0.09-0.11$ \\
4-eqt baro & $\mathrm{SA}+$ Reboud & 0.60 & $0.035-0.076$ \\
4-eqt baro & $\mathrm{KL}+$ Reboud & 0.61 & $0.038-0.085$ \\
4-eqt baro & $\mathrm{KE}+$ Reboud & 0.61 & $0.029-0.078$ \\
4-eqt SG & $\mathrm{SA}+$ Reboud & 0.575 & $0.038-0.085$ \\
4-eqt SG & $\mathrm{KW} \mathrm{SST}$ & 0.595 & $0.030-0.10$ \\
4-eqt Foam & $\mathrm{KW} \mathrm{SST}$ & 0.585 & 0.070 \\
\hline
\end{tabular}




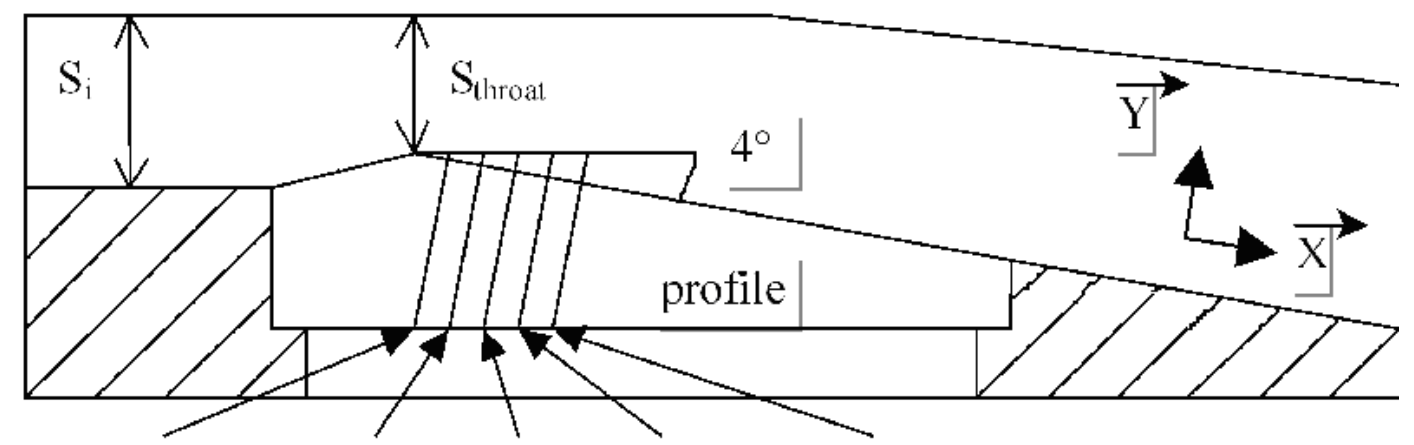

Station 1 Station 2 Station 3 Station 4 Station 5

Figure 1: Schematic view of the $4^{\circ}$ Venturi profile. 


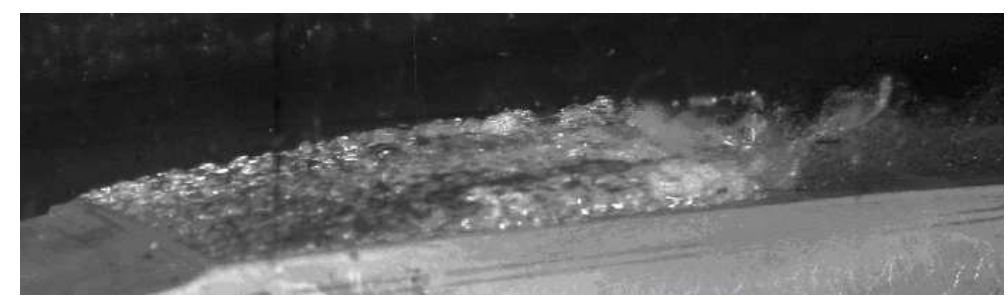

Figure 2: Photograph of the cavitation pocket. 

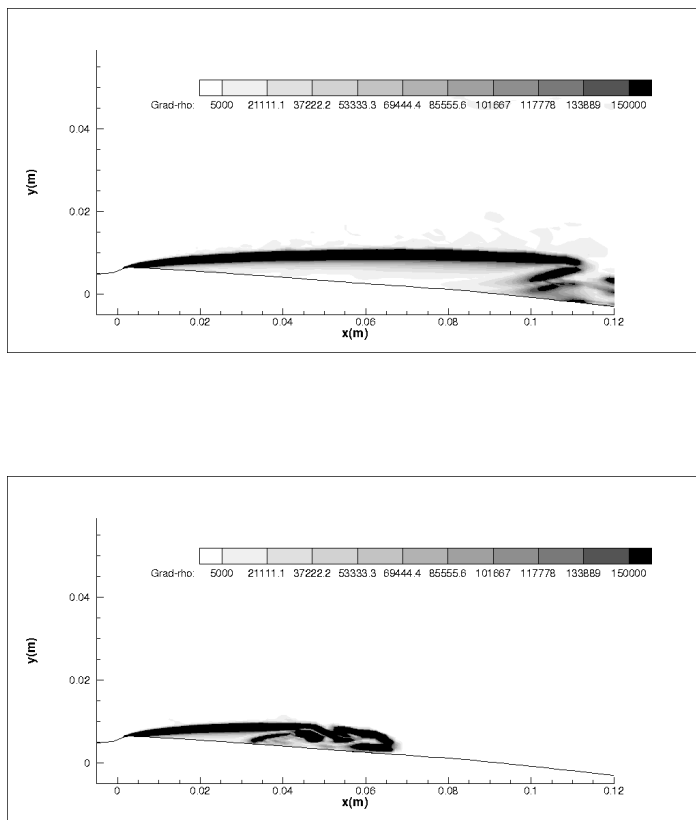

Figure 3: Contours of the density gradient modulus, without limiter (top) and with limiter (bottom). 

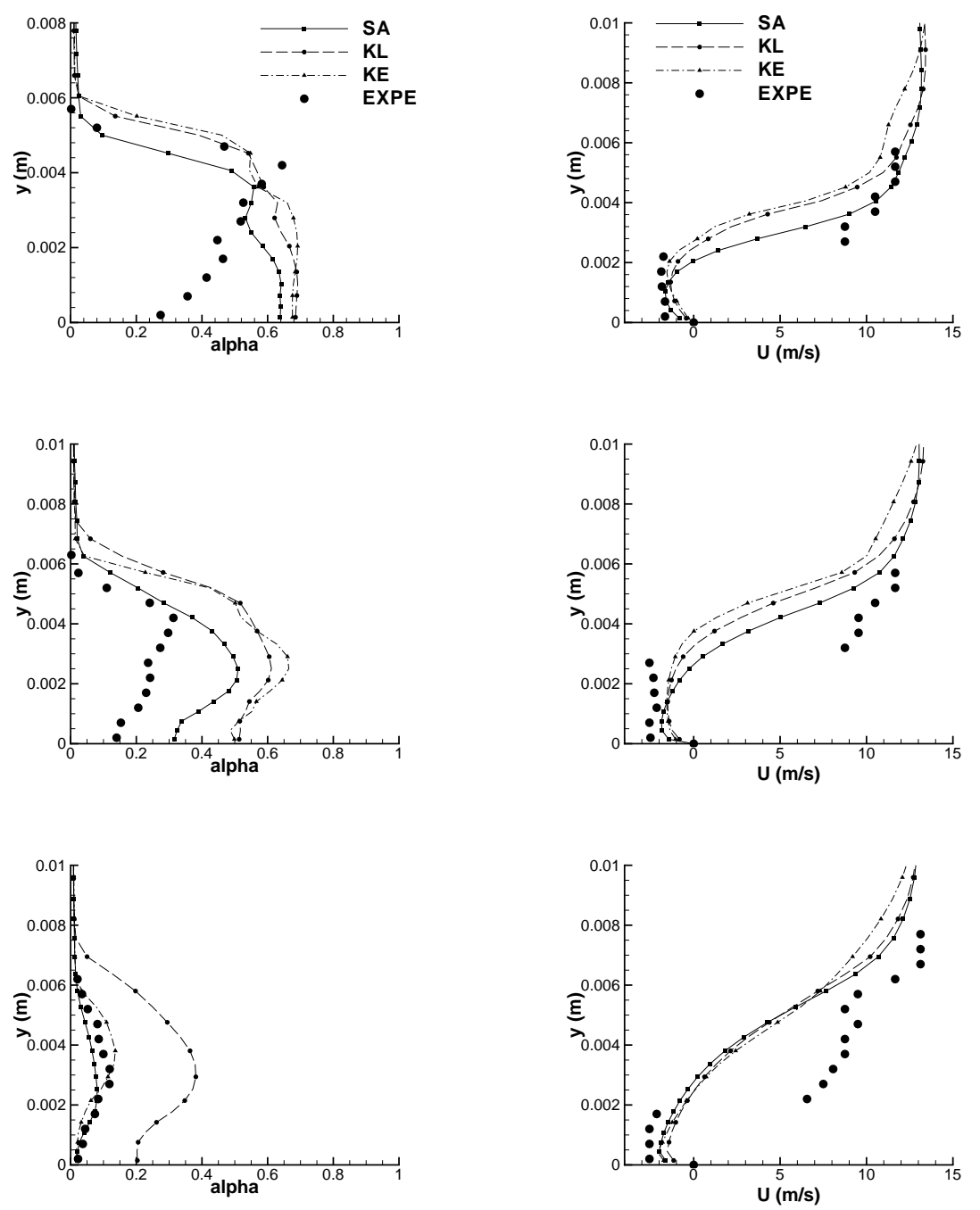

Figure 4: Time-averaged velocity (right) and void ratio (left) profiles from station 3 to 5 , 4-equation barotropic model. 


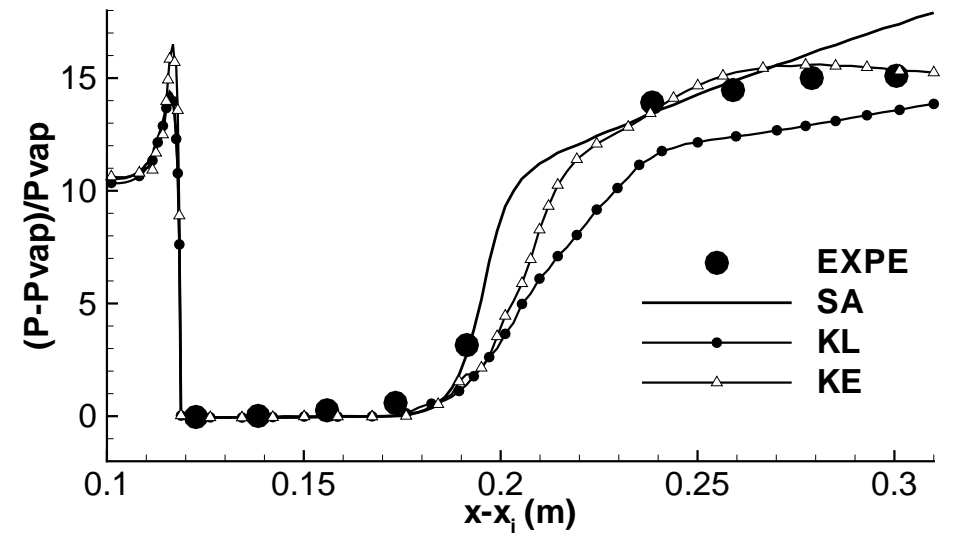

Figure 5: Dimensionless time-averaged wall pressure evolution, 4-equation barotropic model. 


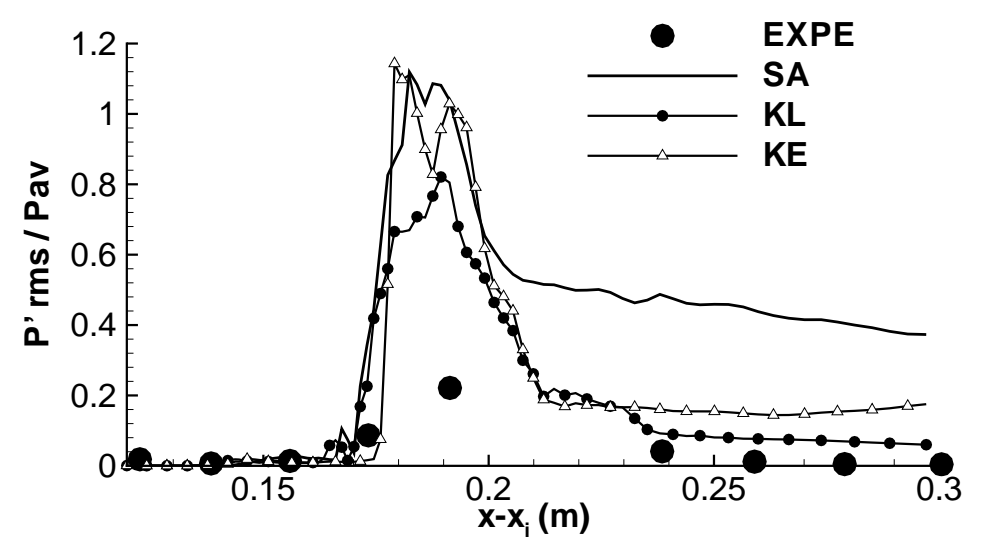

Figure 6: RMS wall pressure fluctuations, 4-equation barotropic model. 


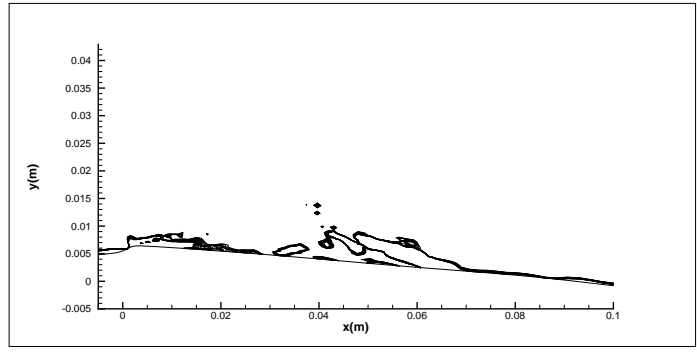

SA model

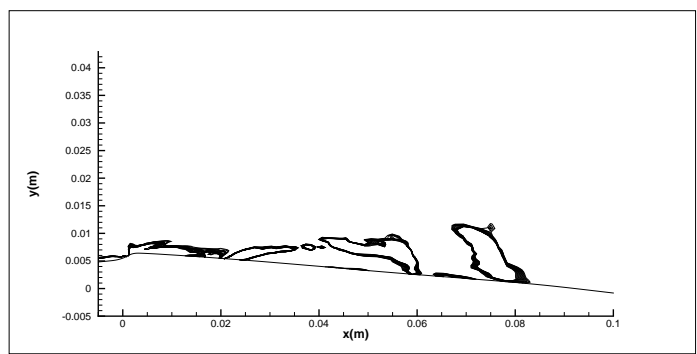

KL model

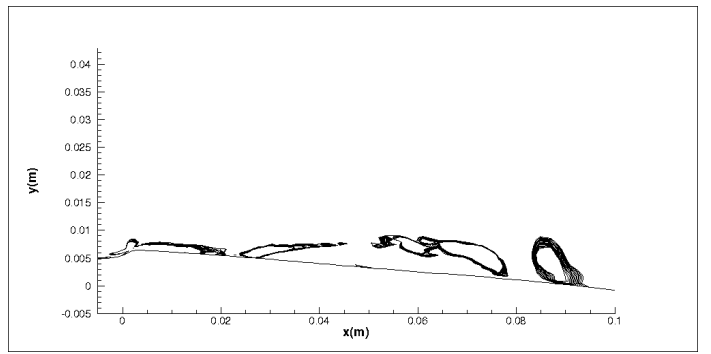

KE model

Figure 7: Isolines of the dimensionless Q-criterion. Turbulence models comparison, 4equation barotropic model. 

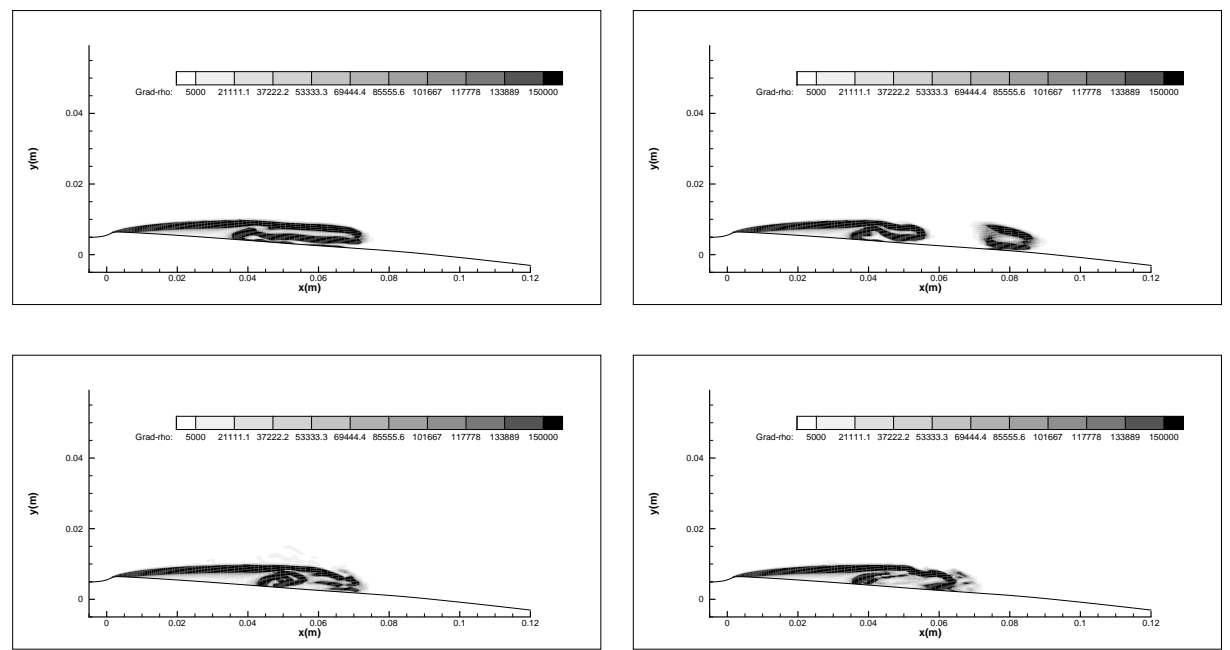

Figure 8: Density gradient modulus $\left(\mathrm{kg} . \mathrm{m}^{-4}\right)$ for 4-eqt SG model (left) and 4-eqt barotropic model (right) 

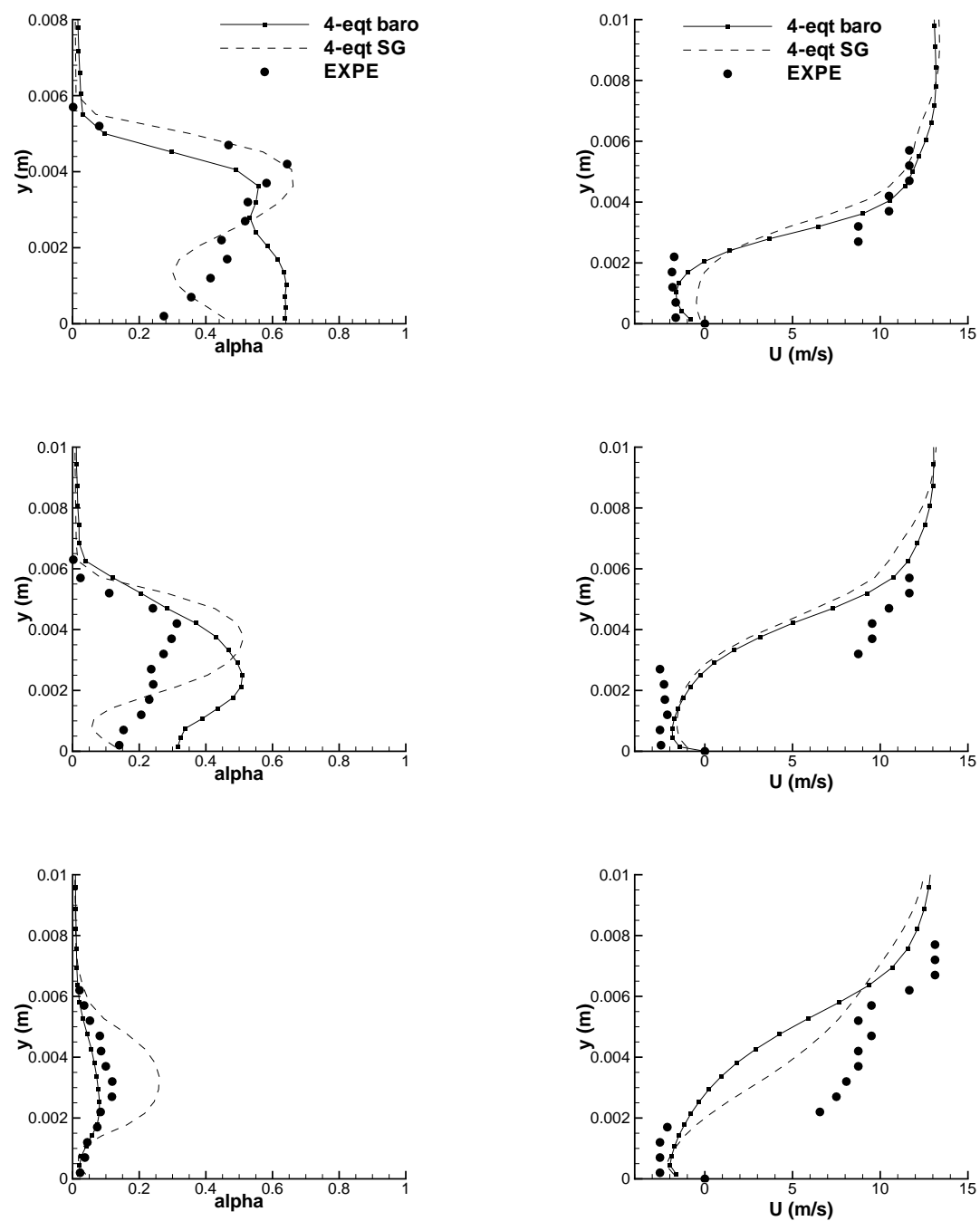

Figure 9: Time-averaged velocity (right) and void ratio (left) profiles from station 3 to 5, 4-equation models comparison. 


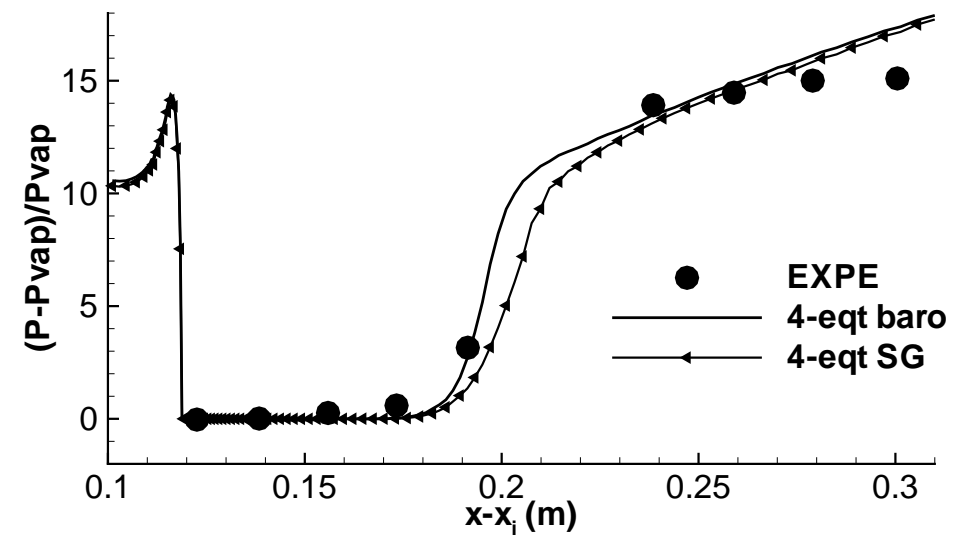

Figure 10: Dimensionless time-averaged wall pressure evolution, 4-equation models comparison. 


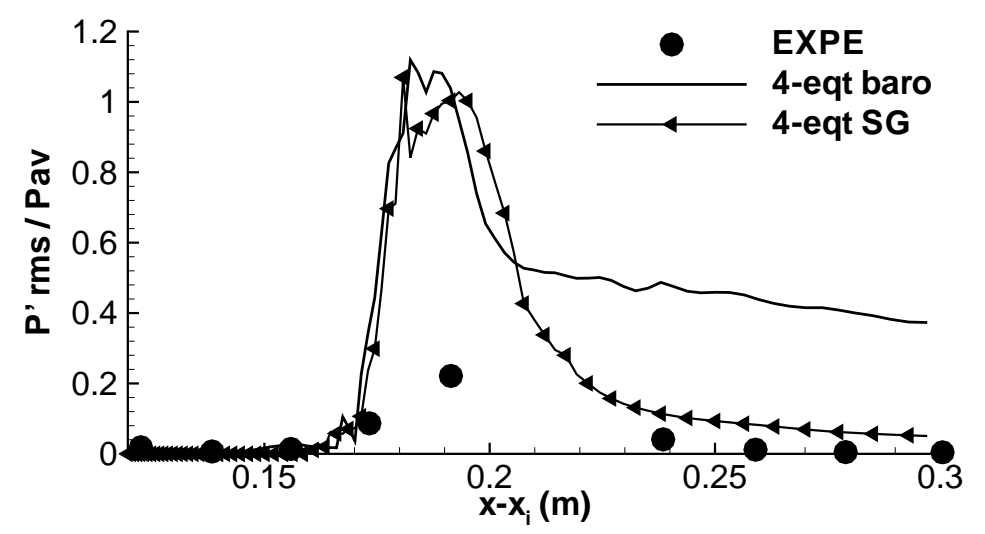

Figure 11: RMS wall pressure fluctuations, 4-equation models comparison. 

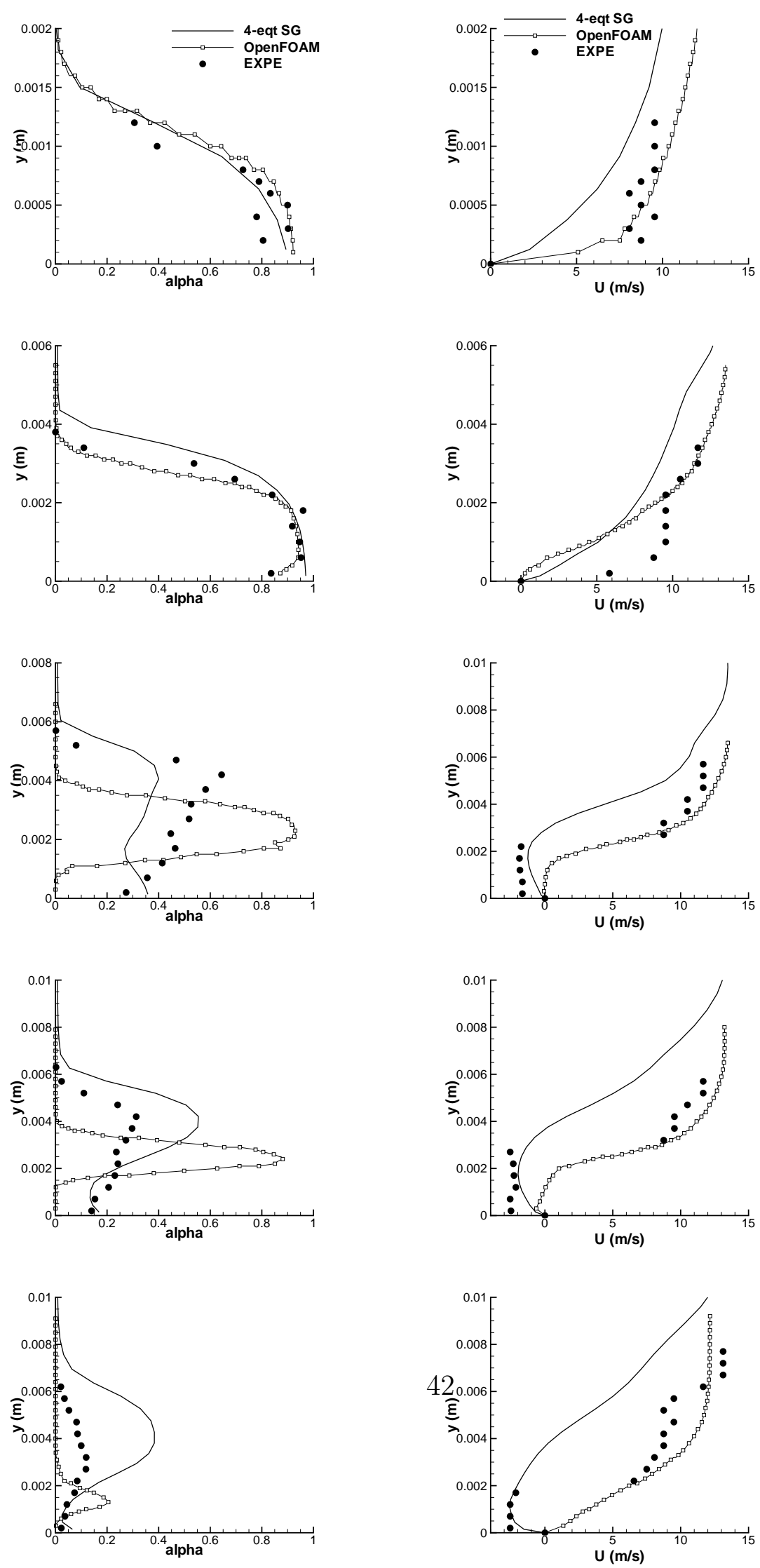

Figure 12: Time-averaged velocity (right) and void ratio (left) profiles from station 1 to 5, LEGI solver versus openFOAM. 


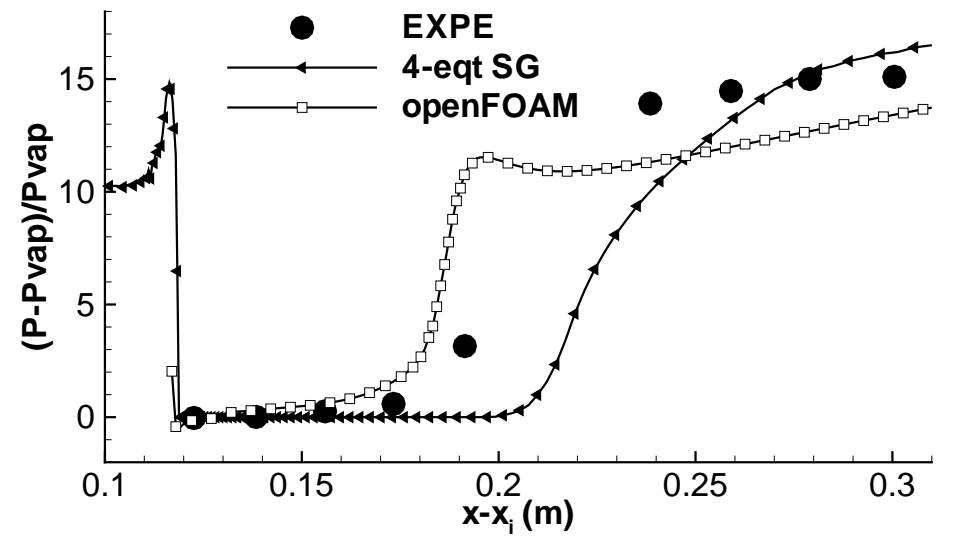

Figure 13: Dimensionless time-averaged wall pressure evolution, LEGI solver versus openFOAM. 


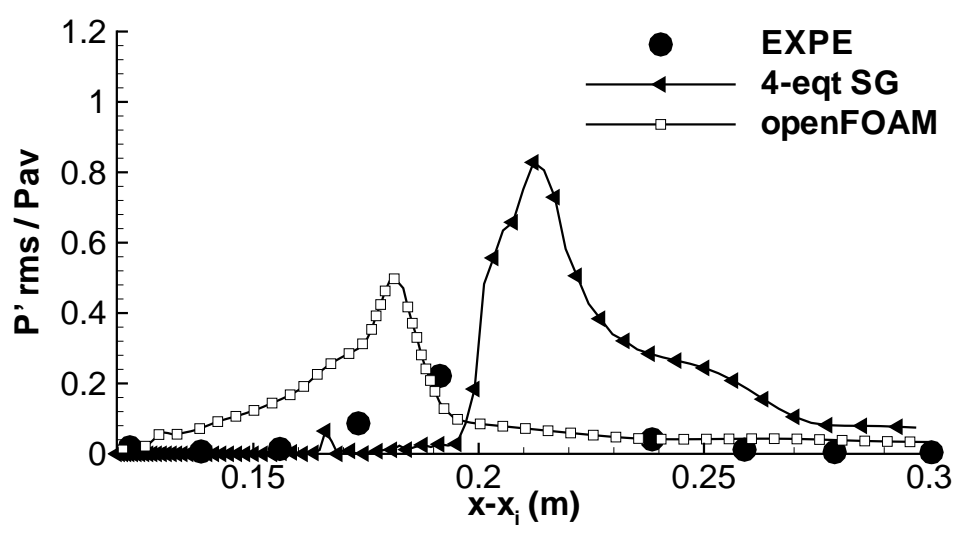

Figure 14: RMS wall pressure fluctuations, LEGI solver versus openFOAM. 